\title{
USO DE RASTROJO DE MAÍZ COMO COBERTURA SUPERFICIAL Y SUS IMPLICACIONES EN LA ECONOMÍA DEL NITRÓGENO EN EL CULTIVO DE MAÍZ
}

\author{
José Luis Zea ${ }^{2}$, M. Osorio ${ }^{2}$, Jorge Bolaños ${ }^{2}$
}

\begin{abstract}
RESUMEN
Uso de rastrojo de maíz como cobertura superficial y sus implicaciones en la economía del nitrógeno en el cultivo de maíz. Con base en los resultados obtenidos en los periodos 89-91, de 1992 a 1995, el Programa Regional de Maíz realizó12 ensayos regionales en los cuales se evaluaron distintos niveles de mantillo $(0,2,5,5$ y $10 \mathrm{t} / \mathrm{ha})$ a varias dosis de nitrógeno (0, 75 y $150 \mathrm{~kg} / \mathrm{ha})$. Algunos de esos ensayos llevan dos o tres añ.os en el mismo sitio. Se encontró una interacción negativa entre efecto neto de mantillo y rendimiento de grano. La respuesta a $\mathrm{N}$ fue positiva en todas las localidades. En todas las localidades hubo ganancia en rendimiento de grano de maíz con cada $\mathrm{kg} \mathrm{N} / \mathrm{ha}$ que se aplicó. En todos los niveles de mantillo, los rendimientos se incrementaron al pasar de 0 a $75 \mathrm{~kg} \mathrm{~N} / \mathrm{ha}$, pero no se obtuvo respuesta al pasar de 75 a $150 \mathrm{~kg} \mathrm{~N} / \mathrm{ha}$. La pudrición de mazorca estuvo asociada con bajo $\mathrm{N}$, más que con niveles altos de mantillo. Los resultados sugieren que para evitar el impacto negativo que produce el uso de mantillo superficial sobre el rendimiento de grano de maíz, se debe considerar la aplicación de una dosis de $\mathrm{N}$ entre $100-150 \mathrm{~kg} / \mathrm{ha}$.
\end{abstract}

\begin{abstract}
The usage of maize wastes as surface cover and its implications in the economy of nitrogen on maize cultivars. Based on results from 89-91,92-95; the Regional Coro Program established 12 regional trials to evaluate severallevels of humus $(0,2.5,5$ and $10 \mathrm{t} / \mathrm{ha})$ at varying levels of Nitrogen $(0.75$ and $150 \mathrm{~kg} / \mathrm{ha})$. Some of these trials have been performed for two or three years at the same site. A negative interaction between net effect of humus and grain yield was found. Response to $\mathrm{N}$ was positive at all localities. At all humus levels, yield increased from 0 to $75 \mathrm{~kg} / \mathrm{ha}$ of N; however, no difference was observed between 75 and 150 $\mathrm{kg} / \mathrm{ha}$ of N. Ear rot was more related to low N levels than to high humus levels. Results suggest that in order to avoid negative impact of superficial humus on corn grain yield, $\mathrm{N}$ must be added at a dose ranging between 100 and $150 \mathrm{~kg} / \mathrm{ha}$.
\end{abstract}

\section{INTRODUCCIÓN}

Cerca del $60 \%$ del maíz que se siembra en Centro América se hace en laderas, con tecnologías que degradan y erosionan rápidamente los suelos (Sosa, 1993) y/o bajo condiciones de humedad que con frecuencia son limitativas para la producción del cultivo por presentarse en el período de floración (INSIVUMEH, 1984). En este contexto, el Programa Regional de Maíz (PRM) investiga desde 1989, nuevas tecnologías que favorezcan la producción sostenida de granos básicos, especialmente maíz, en condiciones marginales y para pequeños y medianos productores. Después de conocer la experiencia de El Salvador, en el área de MetalíoGuaymango (Calderón, 1991), se han realizado una serie de ensayos para determinar el efecto de mantillo (rastrojo) de residuos de la cosecha anterior, especialmente maíz, sobre el rendimiento de maíz (Sosa, 1992; Sosa y Bolaños, 1993).

Violic (1989) manifiesta que las razones expuestas para justificar la preparación del suelo con implementos manuales o mecánicos son hoy todas rebatibles. Las malezas se pueden combatir con herbicidas, los residuos vegetales como mantillo reducen la erosión y mantienen la humedad del suelo, el uso intensivo de arado o rastra deteriora la estructura del suelo, crea el pie de arado y compacta el suelo, etc. También indica que como alternativa al laboreo convencional del suelo han existido desde siempre prácticas conservacionistas, pero en 1940 surge la labranza cero con bases científicas, con el descubrimiento del 2-4 D y otros herbicidas

1 Presentado en la XLII Reunión Anual del PCCMCA en El Salvador., Centroamérica, 1996.

2 CIMMYT-PRM 12 calle 1-25 zona 10, Edificio Géminis 10, Torre Norte, Of. 1606, Guatemala. Tel: (502) (2)353418 - 3534281. 
hormonales, a los cuales se suman en 1950-60 las triazinas (residuales) y más recientemente los desecantes como el paraquat.

Según la Sociedad de Conservación del Suelo de América (1976), labranza de conservación es cualquier sistema que reduce la pérdida de suelo o agua, en comparación con la labranza convencional. Algunos autores consideran que para que realmente sea labranza de conservación debe dejarse por lo menos un $30 \%$ de la superficie del suelo cubierta con mantillo. Johnson (1988), por ejemplo, indica que dejando un 20-30\% de mantillo a la siembra, la erosión se reduce entre 50 y $90 \%$ comparado con un suelo sin protección, aunque menciona que otras variables también participan.

Sin embargo, la aplicación de niveles de mantillo al suelo puede alterar profundamente procesos y propiedades físicas y químicas en el suelo (Bolaños, 1989; Barreto, 1989), debido a cambios que se producen al modificar el sistema de laboreo del suelo. Los cambios más importantes tienen que ver, en este caso, con la mineralización e inmovilización del nitrógeno motivado por la acumulación de la materia orgánica en el perfil del suelo, y principalmente en la capa superficial, bajo labranza cero, cambios ya documentados y que en general indican que la inmovilización de nitrógeno bajo labranza cero es mayor (Barreto, 1989). En muchos casos, más de la mitad del nitrógeno aplicado en el fertilizante se inmoviliza bajo labranza cero en comparación con labranza convencional (Barreta, 1989).

Es necesario resaltar la importancia de la relación $\mathrm{C}: \mathrm{N}$ del rastrojo para entender la mineralización de los residuos vegetales y la disponibilidad de $\mathrm{N}$ bajo labranza cero (Barreto, 1989). La relación C: $\mathrm{N}$ del residuo de maíz-maicillo varía entre 60-80, en comparación con los residuos de leguminosas de cobertura, que tienen $\mathrm{C}: \mathrm{N}$ entre 15-20. Cuando los residuos tienen C:N altos, la descomposición de éstos tiene una demanda fuerte de $\mathrm{N}$, 10 que puede causar una inmovilización del $\mathrm{N}$ disponible en el suelo. Aplicaciones de residuos con C:N inferiores a 20 , resultan en una mineralización neta de $\mathrm{N}$ durante la descomposición de estos. Debido a que los residuos de maíz-maicillo tardan más de seis meses en descomponerse, esto significa una inmovilización importante del $\mathrm{N}$ disponible para el cultivo de maíz. Estudios a largo plazo sugieren que la baja disponibilidad del $\mathrm{N}$ causada por la inmovilización es temporal estableciéndose un nuevo equilibrio en las relaciones de mineralización e inmovilización del suelo (Barreto, 1989).

Experiencias anteriores en el PRM muestran que al aumentar la cantidad de rastrojo (mantillo) disminuye el rendimiento de maíz. Los primeros trabajos se realizaron en El Salvador evaluando niveles de hasta $50 \mathrm{t} / \mathrm{ha}$ de rastrojo. Estudios en 1991 usando niveles de hasta $30 \mathrm{t} /$ ha mostraron que hay efecto negativo sobre rendimiento de grano de maíz y que este es un nivel demasiado alto (Sosa, 1992).

El objetivo principal de estos ensayos fueron enfocar el efecto e interacción de diferentes niveles de mantillo y nitrógeno en diferentes ambientes y años en la región, así como examinar las bases fisiológicas y agronómicas de la posible interacción rastrojo-nitrógeno.

\section{MATERIALES Y MÉTODOS}

En total se establecieron 12 ensayos a través de localidades y años, de la siguiente manera: dos en 1992 (Jutiapa y LaMáquina,Guatemala), seis en 1993 (dos en Guaymango y uno en Texistepeque, El Salvador; Jutiapa y Cuyuta, Guatemala; San Andrés, El Salvador), tres en 1994 (Jutiapa y Cuyuta, Guatemala; San Cristóbal, Nicaragua) y uno en 1995 (Jutiapa, Guatemala). Por país, siete fueron en Guatemala, cuatro en El Salvador y uno en Nicaragua. En algunas localidades (Jutiapa y Cuyuta, Guatemala, 93,94 y 95) los ensayos se establecieron en los mismos sitios en busca de observar efectos a mediano y largo plazo. Casi todos los ensayos fueron establecidos en campos de agricultores. Se usó un arreglo factorial de tratamientos en el diseño de bloques completos al azar, con tres repeticiones por localidad. Los factores evaluados fueron: cantidad de rastrojo (mantillo superficial) con 0,5 y 10 tlha y dosis de nitrógeno con 0,75 y $150 \mathrm{~kg} / \mathrm{ha}$, para un total de nueve tratamientos. En algunas localidades se agregó el nivel 2,5 t/ha (Jutiapa 93, 94 y 95) o se sustituyó este nivel por el de 10 t/ha (Cuyuta 93 y 94). La fertilización fosforada fue uniforme y consistió de $40 \mathrm{~kg} \mathrm{P}_{2} \mathrm{O}_{5} / \mathrm{ha}$, usando triple superfosfato (0-46-0) como fuente.

La unidad experimental total fue de seis surcos de cinco metros de largo, con distancia entre surcos de 0,75 $0,80 \mathrm{~m}$ y entre posturas de 0,40-0,50, dependiendo de la localidad, colocándose dos plantas/postura, para una densidad teórica de 50-53 mil plantas/ha. A la cosecha se tomaron solamente los cuatro surcos centrales. Se usó la variedad de maíz recomendada para cada localidad, tratándose la semilla con insecticida para control de plagas del suelo. Se mantuvo control sobre malezas e insectos del follaje durante el ciclo de cultivo. A la cosecha se midió: número de plantas cosechadas, número de mazorcas totales y podridas, el peso de campo y la humedad del grano. Se tomó también peso de planta (parte aérea) para estimar rendimiento de rastrojo y de biomasa total.

Se realizó un análisis de varianza por localidad y al combinado de las 12 localidades. Se efectuó también análisis de regresión con niveles de rastrojo y nitrógeno. 


\section{RESULTADOS Y DISCUSIÓN}

El análisis de varianza realizado a las principales variables de respuesta, muestra el efecto altamente significativo del nitrógeno, mientras que ni rastrojo ni la interacción de ambos factores tuvo efecto significativo sobre las variables analizadas. La interacción significativa, localidad por $\mathrm{N}$ es esperada, debido al efecto de localidades. No hubo interacción de los otros factores sobre las variables estudiadas.

En términos de efectos netos promedio a través de localidades, años y dosis de N (Cuadro 1), el rendimiento de grano tendio a disminuir conforme se aumento el nivel de rastrojo, lo cual fundamentalmente se debe a que también disminuyó el peso de la mazorca. Esta tendencia ,a través de las diferentes dosis de N, se muestra en la Figura 1. Se nota que cuando no se puso $\mathrm{N}(0 \mathrm{~N})$ el rendimiento de grano fue bajo y decreciente conforme se pasó de 0 a 5 y de 5 a 10 t/ha de rastrojo, mientras que cuando se adicionaron $75 \mathrm{~kg} \mathrm{~N} / \mathrm{ha}$ el rendimiento de incremento en casi el doble, pero sigue siendo decreciente conforme aumento el nivel de rastrojo. Cuando se adicionaron $150 \mathrm{~kg} \mathrm{~N} / \mathrm{ha}$, el rendimiento se incrementó levemente con relación a $75 \mathrm{~kg}$ $\mathrm{N} / \mathrm{ha}$, pero tendió a estabilizarse con respecto a los niveles de rastrojo. Se asume que es hasta esta dosis, o cerca de ella, que se cubre la demanda del cultivo y la de los microorganismos que participan en la descomposición del rastrojo. Sin embargo, no hubo un efecto claro de este factor sobre las variables rendimiento de rastrojo, biomasa ni pudrición de mazorca.
La respuesta al rastrojo, a diferentes dosis de $\mathrm{N}$ (Cuadro 2) muestra que la pérdida de rendimiento de grano de maíz, por cada t/ha de rastrojo aplicada, fue de $16 \mathrm{~kg} / \mathrm{ha}$ grano, cuando no se pone $\mathrm{N}(0 \mathrm{~N})$ y de 51 $\mathrm{kg} / \mathrm{ha}$ cuando se pusieron $75 \mathrm{~kg} \mathrm{~N} / \mathrm{ha}$. Cuando se adicionaron $150 \mathrm{~kg} \mathrm{~N} / \mathrm{ha}$ parece alcanzarse el equilibrio, e indica que la relación $\mathrm{C}: \mathrm{N}$ jugó un papel muy importante en este aspecto (Barreto, 1989), y sólo cuando se pone suficiente $\mathrm{N}$ se logra suplir la demanda de este elemento por fijación y uso en el proceso de descomposición del rastrojo. Sin embargo, se ha encontrado que cuando existe limitación de humedad, como en los años 93 y 94 en localidades como Jutiapa, Cuyuta y San Andrés (Cuadro 2), la aplicación de rastrojo tuvo efectos positivos sobre el rendimiento de maíz, hipotéticamente debido a una mayor retención de humedad en el suelo (Zea, 1995).

Por el contrario, como ya se discutió anteriormente, hubo respuesta positiva a la aplicación de $\mathrm{N}$ (promedio de localidades, años y niveles de rastrojo) en todas las variables, con excepción de pudrición de mazorca. En este sentido, los rendimientos de grano y de rastrojo, la biomasa y el peso de mazorca se incrementaron sustancialmente con la aplicación de $\mathrm{N}$. El rendimiento de grano se incrementó en $1731 \mathrm{~kg} / \mathrm{ha}$, al pasar de 0 a $75 \mathrm{~kg} \mathrm{~N} / \mathrm{ha}(23 \mathrm{~kg}$ grano/ kg N aplicado), en tanto que al pasar de 75 a $150 \mathrm{~kg} \mathrm{~N} / \mathrm{ha}$ el incremento fue de 464 $\mathrm{kg} / \mathrm{ha}$ (6 kg grano/kg N aplicado). Sin embargo, este comportamiento fue variable por cada nivel de rastrojo (Figura 1). Cuando no se puso rastrojo (0 t/ha), hubo un fuerte incremento al pasar de 0 a $75 \mathrm{~kg} \mathrm{~N} / \mathrm{ha}$, pero entre

Cuadro 1. Efecto de nitrógeno y rastrojo sobre las principales variables de respuesta, combinado de 12 localidades. Centro América, 1992-1995.

\begin{tabular}{|c|c|c|c|c|c|c|}
\hline \multirow[t]{2}{*}{ Factor } & \multirow[b]{2}{*}{$\begin{array}{l}\text { Grano } \\
\mathrm{kg} / \mathrm{ha}\end{array}$} & \multirow[b]{2}{*}{$\begin{array}{l}\text { Rast }^{* *} \\
\mathrm{~kg} / \mathrm{ha}\end{array}$} & \multicolumn{4}{|c|}{ Variable de respuesta } \\
\hline & & & $\begin{array}{c}\operatorname{maz}_{\%} \operatorname{pod}^{* * *} \\
\%\end{array}$ & Biomasa & maz/ pta* & $\overline{\text { maz peso }}$ \\
\hline \multicolumn{7}{|l|}{ Nivel Rast (t/ha) } \\
\hline \multicolumn{7}{|l|}{0,0} \\
\hline $2,5^{*}$ & 3758 & 3860 & 11,5 & 7398 & 0,92 & 76,0 \\
\hline 5,0 & 3833 & 3839 & 11,4 & 7300 & 0,97 & 58,5 \\
\hline 10,0 & 3606 & 3620 & 14,8 & 6894 & 0,93 & 75,7 \\
\hline 3573 & 3932 & 11,4 & 7433 & 0.89 & 73,8 & \\
\hline \multicolumn{7}{|l|}{ Nivel N (kg/ha) } \\
\hline 0 & 2365 & 2770 & 15,4 & 4773 & 0,83 & 54,5 \\
\hline 75 & 4096 & 4186 & 11,4 & 8075 & 0,96 & 78,6 \\
\hline 150 & 4560 & 4468 & 10,0 & 8888 & 0,98 & 86,1 \\
\hline
\end{tabular}

* promedio de dos localidades (45 observaciones); ** rastrojo; *** mazorcas podridas; **** mazorcas por planta. 


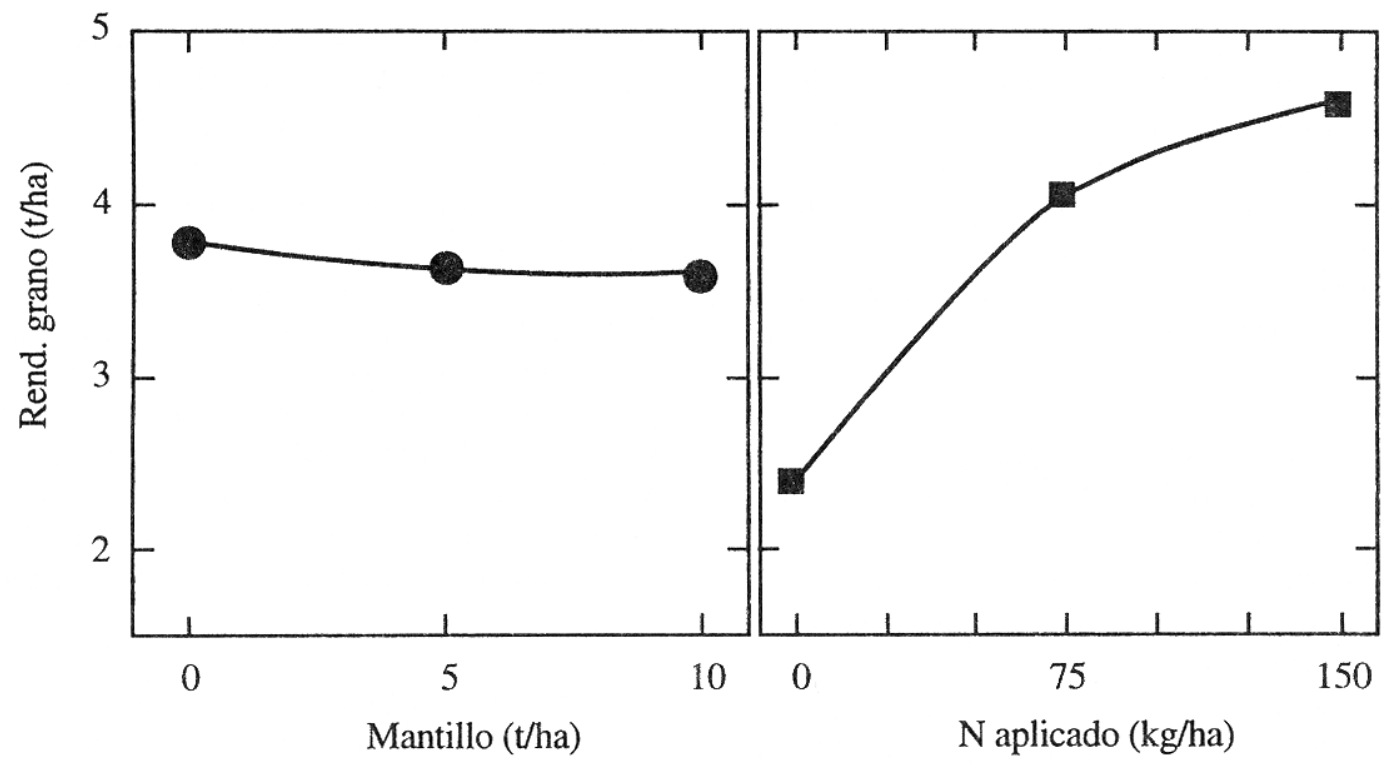

Fig. 1. Interacción Mantillo por Nitrógeno, 11 localidades.

Cuadro 2. Respuesta al rastrojo a diferentes dosis de $\mathrm{N}$ (kg de grano maíz por kg $\mathrm{N}$ aplicado). Centro América, 1992- 1995.

\begin{tabular}{lcccc}
\hline \multicolumn{1}{c}{ Localidad } & \multicolumn{4}{c}{ Dosis de N (kg/ha) } \\
\cline { 2 - 4 } & $\mathbf{0}$ & $\mathbf{7 5}$ & $\mathbf{1 5 0}$ & Combinado \\
\hline 1 La Máquina, G. & $-58^{\mathrm{ns}}$ & $-30^{\mathrm{ns}}$ & $87^{\&}$ & $0^{\mathrm{ns}}$ \\
2 Jutiapa, G. & $-19^{\mathrm{ns}}$ & $-13^{\mathrm{ns}}$ & $63^{\mathrm{ns}}$ & $10^{\mathrm{ns}}$ \\
3 Guaymango, E.S. & $-17^{\mathrm{ns}}$ & $-53^{*}$ & $-9^{\mathrm{ns}}$ & $-27^{\mathrm{ns}}$ \\
4 Guaymango, E.S. & $-21^{\mathrm{ns}}$ & $-14^{\mathrm{ns}}$ & $-18^{\mathrm{ns}}$ & $-59^{\mathrm{ns}}$ \\
5 Texistepeque, ES. & $-56^{\mathrm{ns}}$ & $-90^{\mathrm{ns}}$ & $2^{\mathrm{ns}}$ & $-48^{\mathrm{ns}}$ \\
6 Cuyuta, G. & $-46^{\mathrm{ns}}$ & $-31^{\mathrm{ns}}$ & $49^{\mathrm{ns}}$ & $-9^{\mathrm{ns}}$ \\
7 Jutiapa, G. & $79^{* *}$ & $77^{* *}$ & $88^{\mathrm{n}}$ & $81^{\&}$ \\
8 San Cristóbal, N & $0^{\mathrm{ns}}$ & $-47^{\mathrm{ns}}$ & $19^{\mathrm{ns}}$ & $-9^{\mathrm{ns}}$ \\
9 San Andrés, E.S. & $50^{\mathrm{ns}}$ & $-22^{\mathrm{ns}}$ & $7^{\mathrm{ns}}$ & $11^{\mathrm{ns}}$ \\
10 Jutiapa, G. & $95^{*}$ & $62^{\mathrm{ns}}$ & $37^{\mathrm{ns}}$ & $65^{\mathrm{ns}}$ \\
11 Cuyuta, G. & $150^{\mathrm{ns}}$ & $85^{\mathrm{ns}}$ & $-126^{\mathrm{ns}}$ & $36^{\mathrm{ns}}$ \\
12 Jutiapa, G. & $-77 \&$ & $-39^{* *}$ & $-11^{\mathrm{ns}}$ & $-43^{\mathrm{ns}}$ \\
PROMEDIO. & $-16^{\mathrm{ns}}$ & $-51^{\mathrm{ns}}$ & $3^{\mathrm{ns}}$ & $-21^{\mathrm{ns}}$ \\
\hline
\end{tabular}

$* *=$ significativo al $1 \% ; *=$ significativo al $5 \% ;^{*}=$ significativo al $10 \% ;{ }^{\text {ns }}=$ no significativo.

las dosis 75 y $150 \mathrm{~kg}$ N/ha la respuesta fue mínima, mientras que en los niveles 5 y 10 t/ha, el incremento entre las dosis 0 y $75 \mathrm{~kg} \mathrm{~N} / \mathrm{ha}$ fue menor. Por lo que hay que cubrir las demandas del cultivo y de los microorganismos mineralizadores. Esta misma respuesta se ha observado en otro tipo de ensayos, por lo menos en Guatemala (Zea, 1995), en donde aplicaciones mayores de $75-100 \mathrm{~kg} \mathrm{~N} / \mathrm{ha}$ no muestran mayores incrementos de rendimiento. En términos de promedio general (Cuadro 3) se ganan $15 \mathrm{~kg}$ grano por cada $\mathrm{kg} \mathrm{N} /$ ha aplicado, sin importar el nivel de rastrojo. Sin embargo, debe notarse que esta ganancia es variable por localidad y año, para cada nivel de rastrojo, variando de 4 a 26 en el nivel 0 t/ha, de 1 a 29 en el nivel 5 t/ha, de 4 a 32 en el nivel 10 t/ha y de 3 a 29 en el combinado de las 12 localidades. El incremento en rendimiento estuvo relacionado con el incremento en el número de mazorcas/planta y el peso de mazorca, producido por el $\mathrm{N}$ aplicado, notándose, como en estudios anteriores (Sosa, 1993), que el rastrojo no afecta consistentemente a la variable número de 
Cuadro 3. Respuesta a $\mathrm{N}$ a diferentes niveles de rastrojo (kg de grano maíz por $\mathrm{kg} \mathrm{N}$ aplicado). Centro América, 19921995.

\begin{tabular}{lccccc}
\hline & \multicolumn{5}{c}{ Niveles de rastrojo (t/ha) } \\
\cline { 2 - 6 } Localidad & $\mathbf{0}$ & $\mathbf{2 . 5}$ & $\mathbf{5}$ & $\mathbf{1 0}$ & Combinado \\
\hline 1 La Máquina, G. & $18^{* *}$ & & $23^{* * *}$ & $28^{* * *}$ & $23^{* * *}$ \\
2 Jutiapa, G. & $22^{* *}$ & & $24^{* * *}$ & $27^{* * *}$ & $24^{* * *}$ \\
3 Guaymango, E.S. & $4^{\text {ns }}$ & & $1^{\text {ns }}$ & $4^{* * *}$ & $3^{*}$ \\
4 Guaymango, E.S. & $9^{\text {ns }}$ & & $14^{*}$ & $10^{\text {ns }}$ & $11^{* *}$ \\
5 Texistepeque, ES & $18^{* *}$ & & $20^{* *}$ & $22^{* * *}$ & $20^{* * *}$ \\
6 Cuyuta, G. & $17^{* *}$ & $13^{\text {ns }}$ & $20^{* *}$ & & $17^{* * *}$ \\
7 Jutiapa, G. & $14^{* * *}$ & & $10^{*}$ & $14^{* * *}$ & $12^{* * *}$ \\
8 San Cristóbal, N & $8^{\text {ns }}$ & & $1^{\text {ns }}$ & $9^{*}$ & $5 \&$ \\
9 San Andrés, E.S. & $8^{\text {ns }}$ & & $6^{\text {ns }}$ & $5^{\text {ns }}$ & $6^{*}$ \\
10 Jutiapa, G. & $16^{* * *}$ & $8^{*}$ & $14^{* *}$ & $10^{* *}$ & $12^{* * *}$ \\
11 Cuyuta, G. & $15^{\text {ns }}$ & $11 \mathrm{~ns}$ & $6^{\text {ns }}$ & & $11^{* *}$ \\
12 Jutiapa, G. & $26^{* * *}$ & $30^{* * *}$ & $29^{* * *}$ & $32^{* * *}$ & $29^{* * *}$ \\
PROMEDIO & $15^{* * *}$ & $15^{* *}$ & $14^{* * *}$ & $16^{* * *}$ & $15^{* * *}$ \\
\hline
\end{tabular}

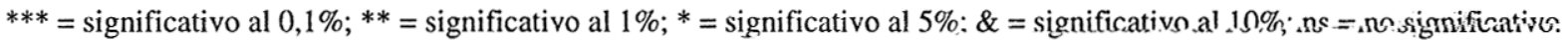

mazorcas/planta o prolificidad (Cuadro 1), a diferencia de otros estrés (Bolaños y Barreto, 1991) a los cuales esta variable es muy sensitiva.

Es importante resaltar que la pudrición de mazorca estuvo más asociada con bajo $\mathrm{N}(0 \mathrm{~N})$ que con niveles altos de rastrojo (Cuadro 2), situación que puede deberse a que el rastrojo utilizado ha estado libre de inóculo o a que las condiciones de los ambientes donde se han conducido los ensayos, no tienen condiciones favorables para esta enfermedad.

\section{LITERATURA CITADA}

BARRETO, J. 1989. Cambios en propiedades químicas, patrones de fertilización y encalamiento en suelos bajo labranza cero. Documento de trabajo CIMMYT-PROCIANDINO. El Batán, México. pp. 43-70.

BOLAÑOS, 1. 1989. Suelos en relación a la labranza de conservación: aspectos físicos. Documento de trabajo CIMMYT-PROCIANDINO. El Batán. México. p. 19-42.

BOLAÑOS, J.; BARRETO, H. 1991. Análisis de los componentes de rendimiento de los ensayos regionales de maíz de 1990. Análisis de los Ensayos Regionales de Agronomía, 1990. Programa Regional de Maíz. Guatemala. pp. 9-25.

CALDERON, F. 1991. Aspectos institucionales de la adaptación y difusión de la labranza de conservación en Metalío-Guaymango, El Salvador. Documento de trabajo Programa Regional de Maíz. Guatemala. pp. 73-83.
INSIVUMEH. 1984. Análisis de la estación lluviosa de Guatemala con fines agrícolas. Departamento de Sistemas Atmosféricos. Sección de Climatología-Agrometeorología. Guatemala. pp 11-12.

JOHNSON, RR 1988. Soil engaging tool effects on surface residue and roughness with chiseltape implements. Soil Sci. Soc. Am. 1. 52:237-243.

SOIL CONSERVATION SOCIETY OF AMERICA. 1976 Resource Conservation Glossary. Ankeny, Iowa.

SOSA, H. 1992. Efecto de la cantidad de mantillo en el rendimiento de los sistemas maíz-sorgo y maíz-frijol, bajo Labranza cero, El Salvador 1991. Síntesis de Resultados Experimentales de 1991. Programa Regional de Maíz. Guatemala. Vol 3. pp. 105-114.

SOSA, H. 1993. Respuesta diferencial del maíz a la labranza de conservación a distintas dosis de nitrógeno. pp. 119123 en Síntesis de Resultados Experimentales del PRM 1992, Guatemala, Vol. 4.

SOSA, H.; J. BOLAÑOS. 1993. Respuesta del maíz-maicillo y maíz-frijol a distintos niveles de mantillo bajo labranza de conservación. Síntesis de Resultados Experimentales del PRM 1992. Guatemala. Vol. 4, pp. 114-118.

VIOLIC, D. 1989. Breve historia de la labranza de conservación. Documento de trabajo CIMMYT-PROCIANDINO. El Batán. México. pp. 1-4.

ZEA, L. 1995. Efecto de dosis de rastrojo y nitrógeno sobre el rendimiento de maíz (Zea mayz L.) en dos localidades de Guatemala. Informe de Resultados 1994, Programa de Maíz, Instituto de Ciencia y Tecnología Agrícolas. Guatemala, pp. 110-115. 